\title{
Backward Stochastic Volterra Integral Equations With Non-Lipschitz Time Delayed Generators
}

\author{
Harouna Coulibaly ${ }^{1} \&$ Auguste Aman ${ }^{1}$ \\ ${ }^{1}$ Laboratoire de Mathématiques Appliquées et Informatique, UFR Mathématiques et Informatique, Université Félix H. \\ Boigny, Abidjan, Côte d'Ivoire \\ Correspondence: Auguste Aman, LMAI, UFR Mathématiques et Informatique, Université Félix H. Boigny, Abidjan, Côte \\ DIvoire. E-mail: augusteaman5@yahoo.fr
}

Received: May 5, 2019 Accepted: May 27, 2019 Online Published: June 3, 2019

doi:10.5539/jmr.v11n3p94 URL: https://doi.org/10.5539/jmr.v11n3p94

\begin{abstract}
In this note, we derive existence and uniqueness results for backward stochastic Volterra integral equations with time delayed generators under non-Lipschitz condition.
\end{abstract}

Keywords: backward stochastic Volterra integral equation, time delayed generator, non-Lipschitz condition

\section{Introduction}

In their works (see Delong \& Imkeller, 2010), Delong and Imkeller introduced a new class of backward stochastic differential equations whose generator depends at each $t \in[0, T]$ on $\left(Y_{t}, Z_{t}\right)=(Y(t+u), Z(t+u))_{-T \leq u \leq 0}$ representing all the past up to $t$ of the process solution. These equations are called backward stochastic differential equations with time delayed generators and took on great importance when Delong in his two works (see Delong, 2012) gives the link between BSDEs with delayed generators and the mathematic formulation of many problems in finance and insurance. For example, he derives that the dynamic of option-based portfolio assurance can be described by the following BSDE with delayed generator:

$$
Y(t)=Y(0)+(Y(T)-Y(0))^{+}-\int_{t}^{T} Z(s) d W(s) .
$$

Since then, the study of BSDEs with delayed generators has been intensified. The work of Dos Reis et al. (Dos Reis \& al., 2012), Zhou and Ren (Zhou \& Ren, 2012), Tuo et al. (Tuo \& al., 2018) and references therein attest this statement. More recently, Coulibaly and Aman (Coulibaly \& Aman, 2019) have studied new class of BSDEs having the form

$$
Y(t)=\xi+\int_{t}^{T} f\left(t, s, Y_{s}, Z_{t, s}\right) d s-\int_{t}^{T} Z(t, s) d W(s)
$$

where $\left(Y_{s}, Z_{t, s}\right)=(Y(s+u), Z(t+u, s+u))_{-T \leq u \leq 0}$ denotes all the past of the solution process until $(t, s) \in[0, T]^{2}$. These BSDEs combine backward stochastic Volterra integral equations (BSVIEs, in short) introduced in (Yong, 2006) and the notion of delayed generators. It is called BSVIEs with delayed generators. This study follows under delayed Lipschitz condition. i.e generator $f$ satisfies the following assumption: there exists a measure of probability $\alpha$ defined on $([-T, 0], \mathcal{B}([-T, 0]))$ and a positive constant $K$, satisfying

$$
\begin{aligned}
& \left|f\left(t, s, y_{s}, z_{t, s}\right)-f\left(t, s, y_{s}^{\prime}, z_{t, s}^{\prime}\right)\right|^{2} \\
\leq & K\left(\int_{-T}^{0}\left|y(s+u)-y^{\prime}(s+u)\right|^{2} \alpha(d u)\right. \\
& \left.+\int_{-T}^{0}\left|z(t+u, s+u)-z^{\prime}(t+u, s+u)\right|^{2} \alpha(d u)\right),
\end{aligned}
$$

for $\mathbb{P} \otimes \lambda$-a.e. $(\omega,(t, s)) \in \Omega \times[0, T]^{2}$ and for any $\left(y_{t}, z_{t, s}\right),\left(y_{t}^{\prime}, z_{t, s}^{\prime}\right) \in L_{-T}^{\infty}(\mathbb{R}) \times L_{-T}^{2}(\mathbb{R})$. They have established existence and uniqueness results when the Lipschitz constant $K$ or the terminal $T$ are small enough. However, for a special class of generators (generators do not depend on $y$ ) and the special delayed measure of probability supported by $[-\delta, 0]$, for $\delta$ 
sufficiently small, the previous results can be extended under an arbitrary Lipschitz constant and any terminal time. On the other hand, we realize that BSVIEs with delayed generator is a suitable tool to formulate some Volterra problem with delay. For example, the notion of expected utility with general memory set by

$$
Y(t)=\mathbb{E}\left(\xi e^{\int_{t}^{T} r(u) d u}+\int_{t}^{T} u\left(\frac{1}{T} \int_{0}^{s} C(u) d u\right) e^{\int_{t}^{s} r(u) d u} d s \mid \mathcal{F}_{t}\right)
$$

where $\frac{1}{T} \int_{0}^{s} C(u) d u$ represents the average of the consumption from 0 to $s$ and $r$ designs the instantaneous discount rate. In more general case, we have

$$
Y(t)=\mathbb{E}\left(\xi e^{\int_{t}^{T} r(u) d u}+\frac{\beta}{T} \int_{t}^{T}\left(\int_{0}^{s} Y(u) d u\right) e^{\int_{t}^{s} r(u) d u} d s \mid \mathcal{F}_{t}\right) .
$$

With the notation of delay framework, the generator of equation (4), which we denote by $f$ can be written as follows:

$$
f\left(t, s, y_{s}, z_{s}\right)=\beta \int_{-T}^{0} y(s+u) e^{\int_{t}^{s} r(v) d v} \alpha(d u)
$$

where $\alpha$ designs the uniform measure of probability on ([-T, 0$], \mathcal{B}([-T, 0]))$. If a instantaneous discount rate $r$ is bounded, then $f$ satisfies the delay Lipschitz condition and according to (Coulibaly \& Aman, 2019), BSVIEs (4) have a unique solution provided that the Lipschitz constant or the terminal time are sufficiently small. On the other hand, let consider the generator $f$ defined by

$$
f\left(t, s, y_{s}, z_{t, s}\right)=\int_{-T}^{0} \frac{\beta}{u+s} y(s+u) e^{s} r(v) d v \alpha(d u)
$$

which appears in expected utility with general memory formulated by

$$
Y(t)=\mathbb{E}\left(\xi e^{\int_{t}^{T} r(u) d u}+\frac{\beta}{T} \int_{t}^{T}\left(\frac{1}{s} \int_{0}^{s} Y(u) d u\right) e^{\int_{t}^{s} r(u) d u} d s \mid \mathcal{F}_{t}\right)
$$

It is clear that $f$ defined in (5) does not satisfy delay Lipschitz condition described by (2). More generally, let consider the function $g$ defined by

$$
g\left(t, s, y_{s}, z_{t, s}\right)=\int_{-T}^{0} \gamma(u)(y(s+u)+z(t+u, s+u)) \alpha(d u),
$$

such that $\gamma$ is neither bounded nor $\alpha$-absolutely integrable on $([-T, 0], \mathcal{B}([-T, 0])$. Then the generator $g$ defined in (6) does not respect a delayed Lipschitz condition stated in (2). Hence Theorem 3.1 appeared in (Coulibaly \& Aman, 2019) can not be used to study a BSVIEs associated to this generator $g$. Better, to our best knowledge there is no results on BSVIEs with non-Lipschitz time delayed generators in litterature.

This paper aims to correct this gap by establishing a result of existence and uniqueness for BSVIEs with non-Lipschitz delayed generator. The rest of this paper is organized as follows. In Section 2, we introduce some fundamental knowledge and assumptions concerning the data of BSVIE (1). Section 3 is devoted to derive existence and uniqueness problems.

\section{Preliminaries}

Let us consider a standard $d$-dimensional Brownian motion $W=(W(t), t \geq 0)$ defined on a probability space $(\Omega, \mathcal{F}, \mathbb{P})$. We denote by $\mathbf{F}=\left(\mathcal{F}_{t}\right)_{t \geq 0}$ the filtration generated by $W$ and augmented by all $\mathbb{P}$-null sets such that the filtered probability space $\left(\Omega, \mathcal{F}_{t}, \mathbf{F}, \mathbb{P}\right)$ satisfies the usual conditions. The Euclidean norm of $\mathbb{R}$ is denoted by $|\cdot|$. Next, for $\beta>0$, we consider the following spaces.

- Let $L^{2}\left(\Omega, \mathcal{F}_{T}, \mathbb{P}\right)$ be the space of $\mathcal{F}_{T}$-measurable random variables $\xi: \Omega \rightarrow \mathbb{R}$ normed by $\|\xi\|_{L^{2}}^{2}=\mathbb{E}\left(|\xi|^{2}\right)$.

- Let $\mathbb{H}_{1}^{\beta}:=\mathcal{H}_{[-T, T]}^{2}(\mathbb{R})$ denote the space of all predictable process $\eta$ with values in $\mathbb{R}$ such that $\mathbb{E}\left[\int_{-T}^{T} e^{\beta s}|\eta(s)|^{2} d s\right]<+\infty$. - Let $\mathbb{H}_{2}^{\beta}:=\mathcal{H}_{[0, T]^{2}}^{2}(\mathbb{R})$ denote the space of all predictable process $\varphi$ with values in $\mathbb{R}$ such that $\mathbb{E}\left[\int_{0}^{T} \int_{0}^{T} e^{\beta s}|\varphi(t, s)|^{2} d s d t\right]<$
$\quad+\infty$ 
- Let $\mathcal{S}^{2}(\mathbb{R})$ denote the space of all predictable process $\eta$ with values in $\mathbb{R}$ such that $\mathbb{E}\left[\sup _{0 \leq s \leq T} e^{\beta s}|\eta(s)|^{2}\right]<+\infty$.

We endowed the spaces $\mathbb{H}_{1}^{\beta}, \mathbb{H}_{2}^{\beta}$ and $\mathcal{S}^{2}(\mathbb{R})$ respectively with the norm $\|\eta\|_{\mathbb{H}_{1}}^{2}=\mathbb{E}\left[\int_{-T}^{T} e^{\beta s}|\eta(s)|^{2} d s\right]$,

$\|\varphi\|_{\mathbb{H}_{2}}^{2}=\mathbb{E}\left[\int_{0}^{T} \int_{0}^{T} e^{\beta s}|\varphi(t, s)|^{2} d s d t\right]$ and $\|\eta\|_{\mathcal{S}^{2}}^{2}=\mathbb{E}\left[\sup _{0 \leq s \leq T} e^{\beta s}|\eta(s)|^{2}\right]$. We also consider this two additive spaces

- Let $L_{-T}^{2}(\mathbb{R})$ denote the space of measurable functions $z:[-T ; 0] \rightarrow \mathbb{R}$ satisfying

$$
\int_{-T}^{0}|z(t)|^{2} d t<+\infty
$$

- Let $L_{-T}^{\infty}(\mathbb{R})$ denote the space of bounded, measurable functions $y:[-T, 0] \rightarrow \mathbb{R}$ satisfying

$$
\sup _{-T \leq t \leq 0}|y(t)|^{2}<+\infty
$$

Before giving our study's framework, let us clarify some notations appearing in this paper. Since BSVIEs considered in this work is a time delay type, we set by $(Y(t), Z(t, s))$, the value of solution process at $(t, s) \in[0, T]^{2}$ and by $\left(Y_{t}, Z_{t, s}\right)=$ $(Y(t+u), Z(t+u, s+u))_{-T \leq u \leq 0}$ the past of this solution until $(t, s)$. Therefore, for each $(t, s) \in[0, T]^{2}$ and almost all $\omega \in \Omega, \quad Y_{t}(\omega)$ and $Z_{t, s}(\omega)$ belong respectively to $L_{-T}^{\infty}(\mathbb{R})$ and $L_{-T}^{2}(\mathbb{R})$.

Let us assume now that our existence and uniqueness result to BSVIEs (1) has been provided under the following assumptions on data.

(A1) $\xi \in L^{2}\left(\Omega, \mathcal{F}_{T}, \mathbb{P}\right)$.

(A2) $f: \Omega \times[0, T] \times L_{-T}^{\infty}(\mathbb{R}) \times L_{-T}^{2}(\mathbb{R}) \rightarrow \mathbb{R}$ is product measurable and $\mathbf{F}$-adapted non-Lipschitz function in the sense that there exists a measure of probability $\alpha$ defined on $([-T, 0], \mathcal{B}([-T, 0]))$ and a non absolute $\alpha$-integrable function $\gamma:[-T, 0] \rightarrow \mathbb{R}$, satisfying

$$
\left|\int_{-T}^{0} \gamma(u) h(u) \alpha(d u)\right| \leq\left|\int_{-T}^{0} \gamma(u) \alpha(d u)\right| \sup _{-T \leq u \leq 0} h(u)
$$

for all function $h:[-T, 0] \rightarrow \mathbb{R}_{+}$, such that

$$
\begin{aligned}
\left|f\left(t, s, y_{s}, z_{t, s}\right)-f\left(t, s, y_{s}^{\prime}, z_{t, s}^{\prime}\right)\right| \leqslant & \int_{-T}^{0} \gamma(u)\left|y(s+u)-y^{\prime}(s+u)\right| \alpha(d u) \\
& +\int_{-T}^{0} \gamma(u)\left|z(t+u, s+u)-z^{\prime}(t+u, s+u)\right| \alpha(d u) .
\end{aligned}
$$

for $\mathbb{P} \otimes \lambda$-a.e. $(\omega, t) \in \Omega \times[0, T]$ and for any $\left(y_{t}, z_{t}\right),\left(y_{t}^{\prime}, z_{t}^{\prime}\right) \in L_{-T}^{\infty}(\mathbb{R}) \times L_{-T}^{2}(\mathbb{R})$,

(A3)

(i) For $t<0$ or $s<0, f(t, s, .,)=$.0 ,

(ii) $\mathbb{E}\left[\int_{[0, T]^{2}}|f(t, s, 0,0)|^{2} d s d t\right]<+\infty$.

To end this section, let deliver these two remarks which explain Assumption (A3).

\section{Remark 2.1}

(a) Assumption (A3)-(i) allows us to take $Y(t)=Y(0)$ and $Z(t, s)=0$ for $t<0$ or $s<0$, as a solution of (1).

(b) The quantity $f(t, s, 0,0)$ in (A3)-(ii) should be understood as a value of the generator $f\left(t, s, y_{s}, z_{t, s}\right)$ at $y_{s}=z_{t, s}=0$.

\section{Main Results}

In this section, we give the main result of this paper which is existence and uniqueness result (Theorem 3.2) for BSVIEs with non Lipschitz time delayed generators. Before, let first derive this needed proposition. 
Proposition 3.1 Assume (A1), (A2) and (A3) hold. Let (Y,Z) belong to $\mathbb{H}_{1} \times \mathbb{H}_{2}$ and satisfy (1). Then $Y \in \mathcal{S}^{2}$.

Proof. Let $(Y, Z)$ be a process belongs in $\mathbb{H}_{1} \times \mathbb{H}_{2}$ and satisfies (1). Applying Itô's formula to $e^{\frac{\beta}{2} t} Y(t), \quad(\beta>0)$, we have

$$
\begin{aligned}
& e^{\frac{\beta}{2} t} Y(t)+\frac{\beta}{2} \int_{t}^{T} e^{\frac{\beta}{2} s} Y(s) d s \\
= & e^{\frac{\beta}{2} T} \xi+\int_{t}^{T} e^{\frac{\beta}{2} s} f\left(t, s, Y_{s}, Z_{t, s}\right) d s-\int_{t}^{T} e^{\frac{\beta}{2} s} Z(t, s) d W(s) .
\end{aligned}
$$

Conditional expectation with respect to $\mathcal{F}_{t}$ taken in each term of previous equality provides

$$
e^{\frac{\beta}{2} t}|Y(t)| \leqslant e^{\frac{\beta}{2} T} \mathbb{E}\left(|\xi| \mid \mathcal{F}_{t}\right)+\mathbb{E}\left(\int_{0}^{T} e^{\frac{\beta}{2} s}\left|f\left(t, s, Y_{s}, Z_{t, s}\right)\right| d s \mid \mathcal{F}_{t}\right) .
$$

Moreover, Doob's inequality with Cauchy-Schwarz's and Jensen's inequalities yield

$$
\mathbb{E}\left[\sup _{0 \leqslant t \leqslant T} e^{\beta t}|Y(t)|^{2}\right] \leq 8 e^{\beta T} \mathbb{E}\left(|\xi|^{2}\right)+8 \mathbb{E}\left(\left.\left|\int_{0}^{T} e^{\beta s / 2}\right| f\left(t, s, Y_{s}, Z_{t, s}\right) d s\right|^{2}\right) .
$$

In view of (A1) the first term of the right side of (8) is finite. Therefore, to prove that $Y$ belongs to $\mathcal{S}^{2}$, it suffice to derive

$$
\mathbb{E}\left[\left(\int_{0}^{T} e^{\beta s / 2}\left|f\left(t, s, Y_{s}, Z_{t, s}\right)\right| d s\right)^{2}\right]<+\infty, \quad \forall t \in[0, T]
$$

which is true if

$$
\mathbb{E}\left[\int_{0}^{T}\left(\int_{0}^{T} e^{\beta s / 2} \mid f\left(t, s, Y_{s}, Z_{t, s}\right) d s\right)^{2} d t\right]<+\infty
$$

To establish (9), let proceed as follows. Applying (A2) together with Fubini's theorem, change of variable, and since $Y(t)=Y(0)$ if $t<0$ and $Z(t, s)=0$ if $t<0$ or $s<0$, we get

$$
\begin{aligned}
& \left(\int_{0}^{T} e^{\beta s / 2}\left|f\left(t, s, Y_{s}, Z_{t, s}\right)\right| d s\right)^{2} \\
\leq & 2\left(\int_{0}^{T} e^{\beta s / 2} \int_{-T}^{0} \int_{-T}^{0} \gamma(u)(|Y(s+u)|+|Z(t+u, s+u)|) \alpha(d u) \alpha(d u) d s\right)^{2} \\
& +2 T \int_{0}^{T} e^{\beta s}|f(t, s, 0,0)|^{2} d s . \\
\leq & 2\left(\int_{-T}^{0} \int_{-T}^{0} e^{-\beta u / 2} \gamma(u) \int_{u}^{T+u} e^{\beta s / 2}(|Y(s)|+|Z(t+u, s)|) d s \alpha(d u) \alpha(d u)\right)^{2} \\
& +2 T \int_{0}^{T} e^{\beta s}|f(t, s, 0,0)|^{2} d s \\
\leq & 2 e^{\beta T}\left(\int_{-T}^{0} \gamma(u) \alpha(d u)\right)^{2}\left[\int_{-T}^{T} e^{\beta s / 2}|Y(s)| d s+\int_{-T}^{0} \int_{0}^{T} e^{\beta s / 2}|Z(t+u, s)| d s \alpha(d u)\right]^{2} \\
& +2 T \int_{0}^{T} e^{\beta s}|f(t, s, 0,0)|^{2} d s \\
\leq & C\left[\int_{-T}^{T} e^{\beta s}|Y(s)|^{2} d s+\int_{-T}^{0} \int_{0}^{T} e^{\beta s}|Z(t+u, s)|^{2} d s \alpha(d u)\right] \\
& +2 T \int_{0}^{T} e^{\beta s}|f(t, s, 0,0)|^{2} d s .
\end{aligned}
$$


So, using again Fubini's theorem, change of variable and recall that $Z(t, s)=0$ if $t<0$ or $s<0$, we obtain

$$
\begin{aligned}
& \mathbb{E}\left[\int_{0}^{T}\left(\int_{0}^{T} e^{\beta s / 2}\left|f\left(t, s, Y_{s}, Z_{t, s}\right)\right| d s\right)^{2} d t\right] \\
\leq & C \mathbb{E}\left[\int_{-T}^{T} e^{\beta s}|Y(s)|^{2} d s+\int_{-T}^{0} \int_{0}^{T} \int_{0}^{T} e^{\beta s}|Z(t+u, s)|^{2} d s d t \alpha(d u)+\int_{[0, T]^{2}} e^{\beta s}|f(t, s, 0,0)|^{2} d s d t\right] \\
\leq & C \mathbb{E}\left[\int_{-T}^{T} e^{\beta s}|Y(s)|^{2} d s+\int_{-T}^{0} \int_{u}^{T+u} \int_{0}^{T} e^{\beta s}|Z(t, s)|^{2} d s d t \alpha(d u)+\int_{[0, T]^{2}} e^{\beta s}|f(t, s, 0,0)|^{2} d s d t\right] \\
\leq & C \mathbb{E}\left[\int_{-T}^{T} e^{\beta s}|Y(s)|^{2} d s+\int_{[0, T]^{2}} e^{\beta s}|Z(t, s)|^{2} d s d t+\int_{[0, T]^{2}} e^{\beta s}|f(t, s, 0,0)|^{2} d s d t\right],
\end{aligned}
$$

which is finite in view of (A3-(ii)) and since $(Y, Z)$ belongs in $\mathbb{H}_{1} \times \mathbb{H}_{2}$.

Theorem 3.2 Assume (A1), (A2) and (A3) hold such that

$$
26 T \max (1, T)\left|\int_{-T}^{0} \gamma(u) \alpha(d u)\right|^{2}<1 .
$$

Then BSVIEs (1) has a unique solution.

Let give these remarks which allow us to compare our result to existing results in order to show its importance.

\section{Remark 3.3}

(i) If in assumption (A2), the function $\gamma$ is bounded or square integrable on $([-T, 0], \mathcal{B}([-T, 0]), \alpha)$, then function $f$ satisfies non-Lipschitz assumed in (Coulibaly \& Aman). In this case, our result coincides with one appears in (Coulibaly \& Aman) (see Theorem 3.1). However, as we stated in introduction, if $\gamma$ is neither bounded nor square integrable on $([-T, 0], \mathcal{B}([-T, 0]), \alpha)$ then the generator $f$ associated does not respect a delayed Lipschitz condition state in (A2). Therefore we can not use Theorem 3.1 appeared in (Coulibaly $\&$ Aman) to study BSVIEs with delayed generator $f$ while our result (Theorem 3.1) remains applicable provided that (11) holds.

(ii) According to (11), if the function $\gamma$ is non negative, then we can find a probability measure $\alpha_{1}$ defined by

$$
\alpha_{1}(d u)=\frac{\gamma(u)}{\int_{-T}^{0} \gamma(u) \alpha(d u)} \alpha(d u)
$$

such that $f$ satisfies Lipschitz condition state in (Coulibaly \& Aman) with $\alpha_{1}$ as a measure of probability.

(iii) If $\gamma$ takes both positive and negative values and is not $\alpha$-absolutely integrable on $([-T, 0], \mathcal{B}([-T, 0])$ ) (which holds in our context) it is impossible to find a measure of probability such as $f$ satisfies the delayed Lipschitz condition with such a measure. This is very essential and reassures that our result is a non trivial generalization of Coulibaly and Aman's work.

Proof. Our method is based on the traditional Picard's iteration. Thereby, for $Y^{0}(s)=Z^{0}(t, s)=0$, let define recursively this sequence of BSVIEs as follows: for $n \in \mathbb{N}$,

$$
Y^{n+1}(t)=\xi+\int_{t}^{T} f\left(t, s, Y_{s}^{n}, Z_{t, s}^{n}\right) d s-\int_{t}^{T} Z^{n+1}(t, s) d W(s), \quad 0 \leqslant t \leqslant T .
$$

\section{(i) Existence}

Step 1: Given $\left(Y^{n}, Z^{n}\right) \in \mathbb{H}_{1} \times \mathbb{H}_{2}$, BSVIEs (12) has a unique solution $\left(Y^{n+1}, Z^{n+1}\right) \in \mathbb{H}_{1} \times \mathbb{H}_{2}$.

Indeed, for a fixed $t \in[0, T]$, let define the process $X_{t}^{n+1}$ by

$$
X_{t}^{n+1}(u)=\mathbb{E}\left(\xi+\int_{t}^{T} f\left(t, s, Y_{s}^{n}, Z_{t, s}^{n}\right) d s \mid \mathcal{F}_{u}\right), u \in[0, T] .
$$

Since according to Proposition 3.1,

$$
\mathbb{E} \int_{t}^{T}\left|f\left(t, s, Y_{s}^{n}, Z_{t, s}^{n}\right)\right|^{2} d s<+\infty,
$$


for $t \in[0, T]$, the process $X_{t}^{n+1}$ is a square integrable $\left(\mathcal{F}_{u}\right)$-martingale. Therefore the martingale representation theorem provides a unique process $Z^{n+1}\left(t\right.$, .) belonging in $\mathbb{H}_{2}$ and satisfying

$$
X_{t}^{n+1}(u)=X_{t}^{n+1}(0)+\int_{0}^{u} Z^{n+1}(t, s) d W(s), u \in[0, T] .
$$

In particular, since

$$
X_{t}^{n+1}(T)=X_{t}^{n+1}(0)+\int_{0}^{T} Z^{n+1}(t, s) d W(s)
$$

we have

$$
X_{t}^{n+1}(u)=X_{t}^{n+1}(T)-\int_{t}^{T} Z^{n+1}(t, s) d W(s) .
$$

But in view of its definition, we have also

$$
X_{t}^{n+1}(T)=\xi+\int_{t}^{T} f\left(t, s, Y_{s}^{n}, Z_{t, s}^{n}\right) d s,
$$

which setting $Y^{n+1}(t)=X_{t}^{n+1}(t)$ leads to

$$
Y^{n+1}(t)=\xi+\int_{t}^{T} f\left(t, s, Y_{s}^{n}, Z_{t, s}^{n}\right) d s-\int_{t}^{T} Z^{n+1}(t, s) d W(s) .
$$

Moreover,

$$
\begin{aligned}
\mathbb{E}\left(\int_{-T}^{T}\left|Y^{n+1}(t)\right|^{2} d t\right) & =\mathbb{E}\left[\int_{-T}^{T}\left|\mathbb{E}\left(\xi+\int_{t}^{T} f\left(t, s, Y_{s}^{n}, Z_{t, s}^{n}\right) d s \mid \mathcal{F}_{t}\right)\right|^{2} d t\right] \\
& \leq \int_{-T}^{T} \mathbb{E}\left(\left|\xi+\int_{t}^{T} f\left(t, s, Y_{s}^{n}, Z_{t, s}^{n}\right) d s\right|^{2}\right) d t \\
& \leq 4 T \mathbb{E}\left(|\xi|^{2}\right)+2 \mathbb{E} \int_{[0, T]^{2}}\left|f\left(t, s, Y_{s}^{n}, Z_{t, s}^{n}\right)\right|^{2} d s d t . \\
& <+\infty,
\end{aligned}
$$

which together with Proposition 3.1 lead that $Y^{n+1}$ belongs to $\mathcal{S}^{2}$ and finally provides that $\left(Y^{n+1}, Z^{n+1}\right)$ solves (12). Step 2: The sequence $\left(Y^{n}, Z^{n}\right)$ converges in $\mathbb{H}_{1} \times \mathbb{H}_{2}$.

For $(t, s) \in[0, T]^{2}$, and in virtue of (12) the process $\left(\bar{Y}^{n}(t), \bar{Z}^{n}(t, s)\right)$ defined by $\bar{Y}^{n}(t)=Y^{n+1}(t)-Y^{n}(t)$ and $\bar{Z}^{n}(t, s)=$ $Z^{n+1}(t, s)-Z^{n}(t, s)$ satisfies equation

$$
\bar{Y}^{n}(t)=\int_{t}^{T} f\left(t, s, Y_{s}^{n}, Z_{t, s}^{n}\right)-f\left(t, s, Y_{s}^{n-1}, Z_{t, s}^{n-1}\right) d s-\int_{t}^{T} \bar{Z}^{n}(t, s) d W(s), \quad 0 \leq t \leq T .
$$

The conditional expectation which respect $\mathcal{F}_{t}$ taken in both side of (14) together with Jensen inequality yield

$$
\left|\bar{Y}^{n+1}(t)\right|^{2} \leq \mathbb{E}\left[\left(\int_{0}^{T}\left|f\left(t, s, Y_{s}^{n}, Z_{t, s}^{n}\right)-f\left(t, s, Y_{s}^{n-1}, Z_{t, s}^{n-1}\right)\right| d s\right)^{2} \mid \mathcal{F}_{t}\right] .
$$

Next, taking respectively integral and expectation in both side, we have by using Fubini's theorem

$$
\int_{-T}^{T}\left|\bar{Y}^{n+1}(t)\right|^{2} d t \leq \mathbb{E}\left[\int_{-T}^{T}\left(\int_{0}^{T} \mid f\left(t, s, Y_{s}^{n}, Z_{t, s}^{n}\right)-f\left(t, s, Y_{s}^{n-1}, Z_{t, s}^{n-1}\right) d s\right)^{2} d t\right] .
$$

Recall again (14), we have

$$
\begin{aligned}
\left|\int_{0}^{T} \bar{Z}^{n+1}(t, s) d W(s)\right| \leq & \int_{0}^{T}\left|f\left(t, s, Y_{s}^{n}, Z_{t, s}^{n}\right)-f\left(t, s, Y_{s}^{n-1}, Z_{t, s}^{n-1}\right)\right| d s+\left|\bar{Y}^{n+1}(0)\right| \\
& +\left|\int_{0}^{t} \bar{Z}^{n+1}(t, s) d W(s)\right|
\end{aligned}
$$


First taking the expectation of both sides and then integrating with respect $t$, it follows respectively from Fubini's theorem, Theorem 2.2 of (Yong, 2006) and (15) that

$$
\begin{aligned}
& \mathbb{E}\left(\int_{[0, T]^{2}}\left|\bar{Z}^{n+1}(t, s)\right|^{2} d s d t\right) \\
\leq & \mathbb{E}\left[4 \int_{0}^{T}\left(\int_{0}^{T}\left|f\left(t, s, Y_{s}^{n}, Z_{t, s}^{n}\right)-f\left(t, s, Y_{s}^{n-1}, Z_{t, s}^{n-1}\right)\right| d s\right)^{2} d t\right. \\
& \left.+4 \int_{-T}^{T}\left|\bar{Y}^{n+1}(t)\right|^{2}+2 \int_{0}^{T} \int_{0}^{t}\left|\bar{Z}^{n+1}(t, s)\right|^{2} d s\right] \\
\leq & \mathbb{E}\left[4 \int_{0}^{T}\left(\int_{0}^{T}\left|f\left(t, s, Y_{s}^{n}, Z_{t, s}^{n}\right)-f\left(t, s, Y_{s}^{n-1}, Z_{t, s}^{n-1}\right)\right| d s\right)^{2} d t\right. \\
& \left.+4 \int_{-T}^{T}\left|\bar{Y}^{n+1}(t)\right|^{2}+4 \int_{0}^{T}\left|\bar{Y}^{n+1}(t)\right|^{2} d t\right] \\
\leq & 12 \mathbb{E}\left[\int_{0}^{T}\left(\int_{0}^{T}\left|f\left(t, s, Y_{s}^{n}, Z_{t, s}^{n}\right)-f\left(t, s, Y_{s}^{n-1}, Z_{t, s}^{n-1}\right)\right| d s\right)^{2} d t\right] .
\end{aligned}
$$

Hence, it follows from (15) and (16) that

$$
\begin{aligned}
& \mathbb{E}\left[\int_{-T}^{T}\left|\bar{Y}^{n+1}(t)\right|^{2} d t\right]+\mathbb{E}\left(\int_{[0, T]^{2}}\left|\bar{Z}^{n+1}(t, s)\right|^{2} d s d t\right) \\
\leq & 13 \mathbb{E}\left[\int_{0}^{T}\left(\int_{0}^{T}\left|f\left(t, s, Y_{s}^{n}, Z_{t, s}^{n}\right)-f\left(t, s, Y_{s}^{n-1}, Z_{t, s}^{n-1}\right)\right| d s\right)^{2} d t\right] .
\end{aligned}
$$

Actually it remains to estimate the second side of inequality (17). For this instance, since $f$ is non-Lipschitz with respect (A2), we have

$$
\begin{aligned}
& \mathbb{E}\left[\int_{0}^{T}\left(\int_{0}^{T}\left|f\left(t, s, Y_{s}^{n}, Z_{t, s}^{n}\right)-f\left(t, s, Y_{s}^{n-1}, Z_{t, s}^{n-1}\right)\right| d s\right)^{2} d t\right] \\
\leq & \mathbb{E}\left[\int_{0}^{T}\left(\int_{0}^{T}\left(\int_{-T}^{0} \int_{-T}^{0} \gamma(u)\left(\left|\bar{Y}^{n}(s+u)\right|+\left|\bar{Z}^{n}(t+u, s+u)\right|\right) \alpha(d u) \alpha(d u)\right) d s\right)^{2} d t .\right.
\end{aligned}
$$

Applied respectively Fubini's theorem, the change of variable and (7) and since $Y^{n}(s)=Y^{n}(0)$ and $Z^{n}(t, s)=0$ for all $s<0$ or $t<0$, we obtain

$$
\begin{aligned}
& \mathbb{E}\left[\int_{0}^{T}\left(\int_{0}^{T}\left(\int_{-T}^{0} \int_{-T}^{0} \gamma(u)\left(\left|\bar{Y}^{n}(s+u)\right|+\left|\bar{Z}^{n}(t+u, s+u)\right|\right) \alpha(d u) \alpha(d u)\right) d s\right)^{2} d t\right] \\
= & \mathbb{E}\left[\int_{0}^{T}\left(\int_{-T}^{0} \int_{-T}^{0} \gamma(u)\left(\int_{0}^{T}\left(\left|\bar{Y}^{n}(s+u)\right|+\left|\bar{Z}^{n}(t+u, s+u)\right|\right) d s\right) \alpha(d u) \alpha(d u)\right)^{2} d t\right] \\
= & \mathbb{E}\left[\int_{0}^{T}\left(\int_{-T}^{0} \int_{-T}^{0} \gamma(u)\left(\int_{u}^{T+u}\left(\left|\bar{Y}^{n}(s)\right|+\left|\bar{Z}^{n}(t+u, s)\right|\right) d s\right) \alpha(d u) \alpha(d u)\right)^{2} d t\right] \\
\leq & 2 T \max (1, T)\left|\int_{-T}^{0} \gamma(u) \alpha(d u)\right|^{2} \mathbb{E}\left[\int_{-T}^{T}\left|\bar{Y}^{n}(t)\right|^{2} d t+\int_{-T}^{0} \int_{u}^{T+u} \int_{0}^{T}\left|\bar{Z}^{n}(t, s)\right|^{2} d s d t \alpha(d u)\right] \\
\leq & 2 T \max (1, T)\left|\int_{-T}^{0} \gamma(u) \alpha(d u)\right|^{2} \mathbb{E}\left[\int_{-T}^{T}\left|\bar{Y}^{n}(t)\right|^{2} d t+\int_{[0, T]^{2}}\left|\bar{Z}^{n}(t, s)\right|^{2} d s d t\right],
\end{aligned}
$$

which provides

$$
\begin{aligned}
& \mathbb{E}\left[\int_{0}^{T}\left(\int_{0}^{T}\left|f\left(s, Y_{s}^{n}, Z_{s}^{n}\right)-f\left(s, Y_{s}^{n-1}, Z_{s}^{n-1}\right)\right| d s\right)^{2} d t\right] \\
& \leq 2 T \max (1, T)\left|\int_{-T}^{0} \gamma(u) \alpha(d u)\right|^{2} \mathbb{E}\left[\int_{-T}^{T}\left|\bar{Y}^{n}(t)\right|^{2} d t+\int_{[0, T]^{2}}\left|\bar{Z}^{n}(t, s)\right|^{2} d s d t\right] .
\end{aligned}
$$


Therefore, in view of (18), (17) becomes

$$
\begin{aligned}
& \mathbb{E}\left[\left.\int_{-T}^{T} \bar{Y}^{n+1}(t)\right|^{2} d t\right]+\mathbb{E}\left(\int_{[0, T]^{2}}\left|\bar{Z}^{n+1}(t, s)\right|^{2} d s d t\right) \\
\leq & 26 T \max (1, T)\left|\int_{-T}^{0} \gamma(u) \alpha(d u)\right| \mathbb{E}\left[\int_{-T}^{T}\left|\bar{Y}^{n}(t)\right|^{2} d t+\int_{[0, T]^{2}}\left|\bar{Z}^{n}(t, s)\right|^{2} d s d t\right]
\end{aligned}
$$

and leads by iterative argument

$$
\begin{aligned}
& \mathbb{E}\left[\int_{-T}^{T}\left|\bar{Y}^{n+1}(t)\right|^{2} d t\right]+\mathbb{E}\left(\int_{[0, T]^{2}}\left|\bar{Z}^{n+1}(t, s)\right|^{2} d s d t\right) \\
\leq & \left(\left.26 T \max (1, T)\left|\int_{-T}^{0} \gamma(u) \alpha(d u)\right|^{2}\right|^{n} \mathbb{E}\left[\int_{-T}^{T}\left|Y^{1}(t)\right|^{2} d t+\int_{[0, T]^{2}}\left|Z^{1}(t, s)\right|^{2} d s d t\right] .\right.
\end{aligned}
$$

Since $26 T \max (1, T)\left|\int_{-T}^{0} \gamma(u) \alpha(d u)\right|^{2}<1$, the second side of the last inequality converges to zero, which leads that $\left(Y^{n}, Z^{n}\right)_{n \geq 1}$ is a Cauchy sequence in the Banach space $\mathbb{H}_{1} \times \mathbb{H}_{2}$ and hence converges to a unique process $(Y, Z) \in \mathcal{S}^{2}(\mathbb{R}) \times \mathcal{H}^{2}(\mathbb{R})$.

Step 3: The process $(Y, Z)$ solves BSVIEs (1).

Since $(Y, Z)$ belongs in $\mathbb{H}_{1} \times \mathbb{H}_{2}$ it follows from Proposition that $Y \in \mathcal{S}^{2}$. By taking the limits for (12), we derive easily that $(Y, Z)$ solves $(1)$.

\section{(ii) Uniqueness}

Let $(Y, Z)$ and $\left(Y^{\prime}, Z^{\prime}\right)$ be two solutions of BSVIEs (1). The process $(\Delta Y(t), \Delta Z(t, s))$ defined by $\Delta Y(t)=Y(t)-Y^{\prime}(t)$ $\left.\Delta Z(t, s)=Z(t, s)-Z^{\prime}(t, s)\right)$ satisfies BSVIEs

$$
\Delta Y(t)=\int_{t}^{T} f\left(t, s, Y_{s}, Z_{t, s}\right)-f\left(t, s, Y_{s}^{\prime}, Z_{t, s}^{\prime}\right) d s-\int_{t}^{T} \Delta Z(t, s) d W(s), 0 \leq t \leq T .
$$

Using the existence proof argument we get

$$
\begin{aligned}
& \mathbb{E}\left[\int_{-T}^{T}|\Delta Y(t)|^{2} d t+\int_{[0, T]^{2}}|\Delta Z(t, s)|^{2} d s d t\right] \\
& \leq 26 T \max (1, T)\left|\int_{-T}^{0} \gamma(u) \alpha(d u)\right|^{2} \mathbb{E}\left[\int_{-T}^{T}|\Delta Y(t)|^{2} d t+\int_{[0, T]^{2}}|\Delta Z(t, s)|^{2} d s d t\right],
\end{aligned}
$$

which since $26 T \max (1, T)\left|\int_{-T}^{0} \gamma(u) \alpha(d u)\right|^{2}<1$, that is satisfied if the horizon time $T$ or the integral of $\gamma$ with respect the measure of probability $\alpha$ on $[-T, 0]$ are small enough, provides

$$
\mathbb{E}\left[\int_{-T}^{T}|\Delta Y(t)|^{2} d t+\int_{[0, T]^{2}}|\Delta Z(t, s)|^{2} d s d t\right]=0 .
$$

Finally, this implies that $\Delta Y(t)=0$ and $\Delta Z(t, s)=0$. The proof is now complete.

\section{Conclusion}

This paper establishes a main result (Theorem 3.2) that gives an alternative to the works of Coulibaly and Aman (see Coulibaly \& Aman, 2019). It will permit us to study a large class of assurance and finance problems whose have been like to the works of Delong (see Delong, 2012), has been modeled by BSVIEs with time delayed generators. On the other hand, we hope to extend, in a future paper, the stochastic control problem studied by Chen and Wu in (Chen \& Wu, 2012), replacing classical BSDEs by BSVIEs with non-Lipschitz delayed generators.

\section{Acknowledgements}

Authors thanks anonymous referees for their appropriate criticisms and suggestions that very helpful to us in developing the final version of this work. 


\section{References}

Chen, L., \& Wu, Z. (2012). Dynamic programming principle for stochastic recursive optimal control problem with delayed systems, ESAIM: COCV 18, 1005-1026. https://doi.org/10.1051/cocv/2011187

Coulibaly, H., \& Aman, A. (2019). Backward stochastic Volterra integral equations with time delayed generators, submitted.

Duffie, D., \& Epstein, L. (1992). Stochastic differential utility, Econometrica, 60, 353-394. https://doi.org/10.2307/2951600

Delong, L. (2012). Applications of time-delayed backward stochastic differential equations to pricing, hedging and portfolio management, Applicationes Mathematicae 39, 463-488. https://doi.org/10.1007/978-1-4471-5331-3_10

Delong, L. (2012). BSDEs with time-delayed generators of a moving average type with applications to non-monotone preferences, Stochastic Models, 28, 281-315. https://doi.org/10.1080/15326349.2012.672281

Delong, L., \& Imkeller, P. (2010). Backward stochastic differential equations with time delayed generators-results and counterexamples, Ann. Appl. Probab. 20(4), 1512-1536. https://doi.org/10.1214/09-AAP663

Delong, L., \& Imkeller, P. (2010). On Malliavin's differentiability of BSDEs with time delay generators driven by Brownian motions and Poisson random measures, Stochastic Processes and their Applications, 120, 1748-1175.

Dos Reis, G., Reveillac, A., \& Zhang, J., (2012). FBSDEs with time delayed generators: $L^{p}$-solutions, differentiability, representation formulas and path regularity. Stochastic Processes and their Application, 121, 2114-2150. https://doi.org/10.1016/j.spa.2011.05.002

Tuo, N., Coulibaly, H., \& Aman, A. (2018). Reflected backward stochastic differential equations with delayed generator, Random Operator and Stochastic Equations, 26(1), 11-22. https://doi.org/10.1515/rose-2018-0002

Wang, Z., \& Zhang, X. (2007) Non-Lipschitz backward stochastic volterra type equations with jumps. Stochastics and Dynamics, 7(4) 479-496. https://doi.org/10.1142/S0219493707002128

Yong J. (2006) Backward stochastic Volterra integral equations and some related problems, Stochastic Processes and their Applications, 116(5), 779-795. https://doi.org/10.1016/j.spa.2006.01.005

Zhou, Q., \& Ren, Y. (2012) Reflected backward stochastic differential equations with time delayed generators, Statistics and Probability Letters, 82, 979-990. https://doi.org/10.1016/j.spl.2012.02.012

\section{Copyrights}

Copyright for this article is retained by the author(s), with first publication rights granted to the journal.

This is an open-access article distributed under the terms and conditions of the Creative Commons Attribution license (http://creativecommons.org/licenses/by/4.0/). 